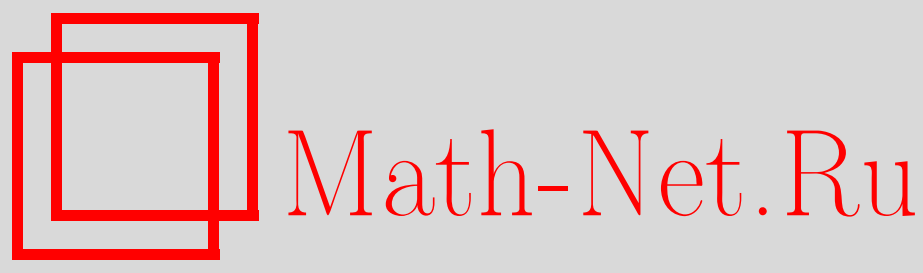

А. А. Андрианов, С. С. Колеватов, Прохождение векторными частицами границы между средой с нарушением пространственной четности и вакуумом, ТМФ, 2013, том 175, номер 3, 357-369

DOI: https://doi.org/10.4213/tmf8473

Использование Общероссийского математического портала Math-Net.Ru подразумевает, что вы прочитали и согласны с пользовательским соглашением http: //www . mathnet.ru/rus/agreement

Параметры загрузки:

IP : 3.81 .55 .215

26 апреля 2023 г., 16:36:02

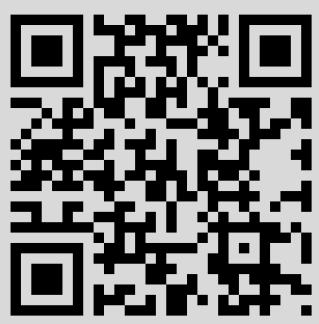




\title{
ПРОХОЖДЕНИЕ ВЕКТОРНЫМИ ЧАСТИЦАМИ ГРАНИЦЫ МЕЖДУ СРЕДОЙ С НАРУШЕНИЕМ ПРОСТРАНСТВЕННОЙ ЧЕТНОСТИ И ВАКУУМОМ
}

\begin{abstract}
Рассмотрена электродинамика с дополнительным действием типа ЧернаСаймонса (электродинамика Керрола-Филда-Джекива) в полупространстве. Исследовано прохождение фотонов и массивных векторных частиц через границу этой среды с вакуумом, в котором действует обычная электродинамика Максвелла. Обнаружены эффекты отражения от границы вплоть до полного отражения при выходе частиц Черна-Саймонса в вакуум и при налетании векторных частиц на эту границу из вакуума.
\end{abstract}

Ключевые слова: нарушение пространственной четности, электродинамика Максвелла-Черна-Саймонса, граничные эффекты.

\section{1. МОДЕЛЬ КЕРРОЛА-ФИЛДА-ДЖЕКИВА С ГРАНИЦЕЙ}

Интерес к возможностям нарушения лоренц-инвариантности и СРТ-четности в Стандартной модели увеличился в последние 20 лет после выхода работы [1]. В ней была рассмотрена модель электродинамики, дополненной лагранжианом ЧернаСаймонса (ЧС), нарушающим четность и ориентированным вдоль постоянного четырехвектора ЧС. На основе анализа излучения удаленных радиогалактик было показано отсутствие этого нарушения на хаббловских расстояниях. Тем не менее спонтанное нарушение лоренц-инвариантности могло бы появиться как эффект среды после конденсации (почти) безмассовых полей аксионов [2]-[5] на расстояниях, сравнимых с размерами звезд или галактик, в частности возле (внутри) очень плотных звезд [6]. Аксионный фон мог бы стать причиной распада высокоэнергетичных фотонов на дилептонные пары [7] и испускания фотонов заряженными частицами [8].

Другая интересная область для наблюдений нарушения четности - физика тяжелых ионов. Не так давно в нескольких экспериментах по столкновению тяжелых ионов был обнаружен аномальный выход лептонных пар [9], [10]. Предполагается, что этот эффект может быть проявлением нарушения четности в столкновении

* Санкт-Петербургский государственный университет, Санкт-Петербург, Россия. E-mail: andrianov@icc.ub.edu; kss2005@list.ru 
ядер благодаря образованию в конечном объеме классического фона (конденсата) псевдоскалярных полей, изосинглетов [11] или нейтральных изотриплетов [12], величина которого зависит от динамики столкновения. В случае аксионоподобного фона в астрофизике и физике тяжелых ионов существование границы между пространством с нарушением четности и вакуумом является существенным. Для аксионных звезд, очевидно, есть граница, где аксионный фон пропадает и фотоны выходят из среды в вакуум. Однако не все фотоны проходят границу, часть отражается, как показано в разделе 2 настоящей работы. Аналогичное явление происходит при облучении фотонами извне звезды с нарушенной четностью, кроме того, дополнительно происходит поворот фазы циркулярно поляризованной волны.

В столкновениях тяжелых ионов выход фотонов/векторных мезонов, распадающихся на лептонные пары, может усиливаться за счет псевдоскалярного фона внутри горячего адронного шара - файербола. Такой фон усредненно описывается аксиальным химическим потенциалом, соответствующим вектору ЧС, направленному вдоль временно́й оси, в то время как граница адронного шара является пространственной. Этот случай исследован в разделе 3 настоящей работы. В обоих случаях обнаружены области энергий векторных мезонов в среде, при которых происходит полное внутреннее отражение.

Таким образом, расчет влияния аксионного/пионного фона в ограниченном объеме на распространение фотонов и векторных мезонов через границу в вакуум и обратно, может стать основой для нахождения областей пространства с нарушением четности.

Описание модели Керрола-Филда-Джекива мы начнем с плотности лагранжиана векторного поля в присутствии аксионного фона:

$$
\mathcal{L}=-\frac{1}{4} F^{\alpha \beta}(x) F_{\alpha \beta}(x)-\frac{1}{4} F^{\mu \nu}(x) \widetilde{F}_{\mu \nu}(x) a_{\mathrm{cl}}(x)+\frac{1}{2} m^{2} A_{\nu}(x) A^{\nu}(x),
$$

где $A_{\mu}$ и $a_{\mathrm{cl}}$ обозначают векторное и фоновое псевдоскалярное поля соответственно, $\widetilde{F}^{\mu \nu}=\varepsilon^{\mu \nu \rho \sigma} F_{\rho \sigma} / 2$ - дуальная напряженность поля. Мы включили массовый член для векторного поля, чтобы рассчитать влияние нарушения четности на образование векторных мезонов $(\rho, \omega, \ldots)$ в столкновении тяжелых ионов [11] и обеспечить непротиворечивость динамики фотонов в случае лагранжиана ЧС [13] с времениподобным вектором ЧС.

Рассмотрим медленно меняющееся фоновое поле вида

$$
a_{\mathrm{cl}}(x)=\zeta_{\lambda} x^{\lambda} \theta(-\zeta \cdot x)
$$

где $\theta(\cdot)$ - функция Хевисайда, в которой аргументом является постоянный четырехвектор $\zeta^{\mu}$ с размерностью массы. При таком выборе лоренц-инвариантность будет нарушена в полупространстве Минковского $\zeta \cdot x<0$. Из формулы $(2)$, используя теорему Гаусса и интегрируя по частям, мы можем вывести уравнения поля из эквивалентного лагранжиана

$$
\mathcal{L}=-\frac{1}{4} F^{\alpha \beta}(x) F_{\alpha \beta}(x)+\frac{1}{2} \zeta_{\mu} A_{\nu}(x) \widetilde{F}^{\mu \nu}(x) \theta(-\zeta \cdot x)+\frac{m^{2}}{2} A_{\nu}(x) A^{\nu}(x)
$$


Соответствующие уравнения для поля имеют вид

$$
\begin{aligned}
\square A^{\nu}(x)+m^{2} A^{\nu}(x) & =\varepsilon^{\nu \alpha \rho \sigma} \zeta_{\alpha} \partial_{\rho} A_{\sigma}(x), & & \zeta \cdot x<0, \\
\square A^{\nu}(x)+m^{2} A^{\nu}(x) & =0, & & \zeta \cdot x>0 .
\end{aligned}
$$

Решим эти уравнения в случае $\zeta \cdot x<0$. Напомним вид киральных векторов поляризации для векторного поля Максвелла-Черна-Саймонса (МЧС), которые строятся при помощи проектора на плоскость, поперечную векторам $k_{\mu}, \zeta_{\nu}[13]$ :

$$
\begin{aligned}
S_{\lambda}^{\nu} & \equiv \delta_{\lambda}^{\nu} \mathrm{D}+k^{\nu} k_{\lambda} \zeta^{2}+\zeta^{\nu} \zeta_{\lambda} k^{2}-\zeta \cdot k\left(\zeta_{\lambda} k^{\nu}+\zeta^{\nu} k_{\lambda}\right) \\
\mathrm{D} & \equiv(\zeta \cdot k)^{2}-\zeta^{2} k^{2}=\frac{1}{2} S_{\nu}^{\nu} .
\end{aligned}
$$

Используя последнее равенство, можно получить соотношени е

$$
S^{\mu \lambda} \varepsilon_{\lambda \nu \alpha \beta} \zeta^{\alpha} k^{\beta}=\mathrm{D} \varepsilon_{\nu \alpha \beta}^{\mu} \zeta^{\alpha} k^{\beta}
$$

Теперь, следуя нашей цели, удобно ввести два ортонормированных одномерных эрмитовых проектора

$$
\boldsymbol{\pi}_{ \pm}^{\mu \nu} \equiv \frac{S^{\mu \nu}}{2 \mathrm{D}} \pm \frac{i}{2} \varepsilon^{\mu \nu \alpha \beta} \zeta_{\alpha} k_{\beta} \mathrm{D}^{-1 / 2}=\left(\boldsymbol{\pi}_{ \pm}^{\nu \mu}\right)^{*}=\left(\boldsymbol{\pi}_{\mp}^{\mu \nu}\right)^{*}, \quad \mathrm{D}>0
$$

Два киральных вектора поляризации для векторного поля МЧС могут быть построены из постоянных тетрад $\epsilon_{\nu}$ :

$$
\varepsilon_{ \pm}^{\mu *}(k) \equiv \boldsymbol{\pi}_{ \pm}^{\mu \lambda} \epsilon_{\mu}
$$

Их свойства подробно описаны в работе [13].

Чтобы получить нормальные моды распространения поля МЧС, введем кинетическую эрмитову $(4 \times 4)$-матрицу $\mathbb{K}$ с элементами

$$
K_{\lambda \nu} \equiv g_{\lambda \nu}\left(k^{2}-m^{2}\right)+i \varepsilon_{\lambda \nu \alpha \beta} \zeta^{\alpha} k^{\beta}, \quad K_{\lambda \nu}=K_{\nu \lambda}^{*}
$$

Общее решение уравнений свободного поля (4) при $\zeta \cdot x<0$ получаем из соотношений (7), (8):

$$
\begin{aligned}
K_{\nu}^{\mu} \varepsilon_{ \pm}^{\nu}(k) & =\left[\delta_{\nu}^{\mu}\left(k^{2}-m^{2}\right)+\sqrt{\mathrm{D}}\left(\boldsymbol{\pi}_{+\nu}^{\mu}-\boldsymbol{\pi}_{-\nu}^{\mu}\right)\right] \varepsilon_{ \pm}^{\nu}(k)= \\
& =\left(k^{2}-m^{2} \pm \sqrt{\mathrm{D}}\right) \varepsilon_{ \pm}^{\mu}(k) .
\end{aligned}
$$




\section{2. КЛАССИЧЕСКИЕ РЕШЕНИЯ ДЛЯ ПРОСТРАНСТВЕННОПОДОБНОГО ВЕКТОРА ЧС}

Рассмотрим случай пространственного вектора ЧС $\zeta_{\mu}=\left(0,-\zeta_{x}, 0,0\right)$. Чтобы решить классические уравнения Эйлера-Лагранжа (4), введем векторы $\hat{k}=\left(\omega, k_{2}, k_{3}\right)$, $\hat{x}=\left(x_{0}, x_{2}, x_{3}\right)$ и их скалярное произведение $\hat{k} \cdot \hat{x}=-\omega x_{0}+k_{2} x_{2}+k_{3} x_{3}$. С помощью преобразования Фурье по выбранным координатам нетрудно решить уравнение для $A_{1}$ во всем пространстве:

$$
\tilde{A}_{1}=\tilde{u}_{1 \rightarrow}\left(\omega, k_{2}, k_{3}\right) e^{i k_{10} x_{1}}+\tilde{u}_{1 \leftarrow}\left(\omega, k_{2}, k_{3}\right) e^{-i k_{10} x_{1}},
$$

где $k_{10}^{2}=\omega^{2}-m^{2}-k_{\perp}^{2}, k_{\perp}^{2}=k_{2}^{2}+k_{3}^{2}$, а стрелки $\rightarrow, \leftarrow$ показывают направление движения частицы.

Рассмотрим систему (4) для оставшихся компонент и проведем преобразование Фурье по координатам $\hat{x}$ :

$$
\begin{aligned}
& \left(-\omega^{2}+m^{2}+k_{\perp}^{2}\right) \tilde{A}_{0}-\partial_{1}^{2} \tilde{A}_{0}=i \zeta_{x} \theta\left(-x_{1}\right)\left(k_{2} \tilde{A}_{3}-k_{3} \tilde{A}_{2}\right), \\
& \left(-\omega^{2}+m^{2}+k_{\perp}^{2}\right) \tilde{A}_{2}-\partial_{1}^{2} \tilde{A}_{2}=-i \zeta_{x} \theta\left(-x_{1}\right)\left(k_{3} \tilde{A}_{0}+\omega \tilde{A}_{3}\right), \\
& \left(-\omega^{2}+m^{2}+k_{\perp}^{2}\right) \tilde{A}_{3}-\partial_{1}^{2} \tilde{A}_{3}=i \zeta_{x} \theta\left(-x_{1}\right)\left(\omega \tilde{A}_{2}+k_{2} \tilde{A}_{0}\right) .
\end{aligned}
$$

Эта система верна во всем пространстве. В случае $x_{1}>0$ получается решение уравнений Максвелла в вакууме, а при $x_{1}<0$ решение описывает электродинамику MЧC:

$$
\tilde{A}_{\nu}= \begin{cases}\tilde{u}_{\nu \rightarrow}\left(\omega, k_{2}, k_{3}\right) e^{i k_{10} x_{1}}+\tilde{u}_{\nu \leftarrow}\left(\omega, k_{2}, k_{3}\right) e^{-i k_{10} x_{1}}, & x_{1}>0, \\ \sum_{A}\left[\tilde{v}_{\nu A \rightarrow}\left(\omega, k_{2}, k_{3}\right) e^{i k_{1 A} x_{1}}+\tilde{v}_{\nu A \leftarrow}\left(\omega, k_{2}, k_{3}\right) e^{-i k_{1 A} x_{1}}\right], & x_{1}<0 .\end{cases}
$$

Здесь первый индекс $\nu=0,2,3$ у функции $\tilde{v}$ обозначает соответствующую компоненты $A_{\nu}$, второй индекс $A$ отвечает за различные законы дисперсии $k_{1}$ для поляризаций $\mathrm{L},+,-$. Ниже приведены законы дисперсии для различных поляризаций:

$$
\begin{aligned}
& k_{1 \mathrm{~L}}=k_{10}=\sqrt{\omega^{2}-m^{2}-k_{\perp}^{2}}, \\
& k_{1+}=\sqrt{\omega^{2}-m^{2}-k_{\perp}^{2}+\zeta_{x} \sqrt{\omega^{2}-k_{\perp}^{2}}} \\
& k_{1-}=\sqrt{\omega^{2}-m^{2}-k_{\perp}^{2}-\zeta_{x} \sqrt{\omega^{2}-k_{\perp}^{2}}} .
\end{aligned}
$$

Кроме того, $v$ удовлетворяют следующим условиям:

$$
\begin{array}{ll}
\tilde{v}_{2+\leftrightarrows}=\frac{k_{2} k_{3}-i \omega \sqrt{\omega^{2}-k_{\perp}^{2}}}{\omega^{2}-k_{2}^{2}} \tilde{v}_{3+\leftrightarrows}, & \tilde{v}_{2-\leftrightarrows}=\frac{k_{2} k_{3}+i \omega \sqrt{\omega^{2}-k_{\perp}^{2}}}{\omega^{2}-k_{2}^{2}} \tilde{v}_{3-\leftrightarrows}, \\
\tilde{v}_{0+\leftrightarrows}=-\frac{i\left(k_{3} \tilde{v}_{2+\leftrightarrows}-k_{2} \tilde{v}_{3+\leftrightarrows}\right)}{\sqrt{\omega^{2}-k_{\perp}^{2}}}, & \tilde{v}_{0-\leftrightarrows}=\frac{i\left(k_{3} \tilde{v}_{2-\leftrightarrows}-k_{2} \tilde{v}_{3-\leftrightarrows}\right)}{\sqrt{\omega^{2}-k_{\perp}^{2}}}, \\
\tilde{v}_{2 L \leftrightarrows}=\frac{k_{2}}{k_{3}} \tilde{v}_{3 L \leftrightarrows}, & \tilde{v}_{0 L \leftrightarrows}=-\frac{\omega}{k_{3}} \tilde{v}_{3 L \leftrightarrows .}
\end{array}
$$


Итак, мы имеем решения в обоих полупространствах, и теперь нам надо склеить их на границе. Проинтегрируем уравнения (12) по переменной $x_{1}$ от $-\varepsilon$ до $\varepsilon$. Это даст нам граничные условия [14]

$$
\begin{aligned}
& k_{10}^{2}\left(-\frac{\tilde{u}_{0 \rightarrow}-\tilde{u}_{0 \leftarrow}}{i k_{10}}+\sum_{A} \frac{\tilde{v}_{0 A \rightarrow}-\tilde{v}_{0 A \leftarrow}}{i k_{1 A}}\right)= \\
& =-i \zeta_{x} \sum_{A}\left(k_{2} \frac{\tilde{v}_{3 A \rightarrow}-\tilde{v}_{3 A \leftarrow}}{i k_{1 A}}-k_{3} \frac{\tilde{v}_{2 A \rightarrow}-\tilde{v}_{2 A \leftarrow}}{i k_{1 A}}\right) \text {, } \\
& k_{10}^{2}\left(-\frac{\tilde{u}_{2 \rightarrow}-\tilde{u}_{2 \leftarrow}}{i k_{10}}+\sum_{A} \frac{\tilde{v}_{2 A \rightarrow}-\tilde{v}_{2 A \leftarrow}}{i k_{1 A}}\right)= \\
& =i \zeta_{x} \sum_{A}\left(k_{3} \frac{\tilde{v}_{0 A \rightarrow}-\tilde{v}_{0 A \leftarrow}}{i k_{1 A}}+\omega \frac{\tilde{v}_{3 A \rightarrow}-\tilde{v}_{3 A \leftarrow}}{i k_{1 A}}\right), \\
& k_{10}^{2}\left(-\frac{\tilde{u}_{3 \rightarrow}-\tilde{u}_{3 \leftarrow}}{i k_{10}}+\sum_{A} \frac{\tilde{v}_{3 A \rightarrow}-\tilde{v}_{3 A \leftarrow}}{i k_{1 A}}\right)= \\
& =-i \zeta_{x} \sum_{A}\left(\omega \frac{\tilde{v}_{2 A \rightarrow}-\tilde{v}_{2 A \leftarrow}}{i k_{1 A}}+k_{2} \frac{\tilde{v}_{0 A \rightarrow}-\tilde{v}_{0 A \leftarrow}}{i k_{1 A}}\right) .
\end{aligned}
$$

Вклады в амплитуду в правом полупространстве $\left(u_{\mu}\right)$, происходящие из амплитуд разных поляризаций левого полупространства независимы: $u_{\mu}=u_{\mu}^{(\mathrm{L})}+u_{\mu}^{(+)}+u_{\mu}^{(-)}$.

В случае $A=\mathrm{L}$ правые части этой системы равны нулю в силу условий (15), поэтому

$$
\tilde{u}_{\nu \rightarrow}^{(\mathrm{L})}-\tilde{u}_{\nu \leftarrow}^{(\mathrm{L})}=\tilde{v}_{\nu \mathrm{L} \rightarrow}-\tilde{v}_{\nu \mathrm{L} \leftarrow}
$$

При $A= \pm$, используя соотношения между $v_{ \pm}$в формулах $(16)$, находим следующие условия:

$$
\tilde{u}_{\nu \rightarrow}^{( \pm)}-\tilde{u}_{\nu \leftarrow}^{( \pm)}=\frac{\left(\tilde{v}_{\nu \pm \rightarrow}-\tilde{v}_{\nu \pm \leftarrow}\right) k_{1+}}{k_{10}} .
$$

Кроме того, все вклады в векторное поле $A$ непрерывны:

$$
\tilde{u}_{\nu \rightarrow}^{(A)}+\tilde{u}_{\nu \leftarrow}^{(A)}=\tilde{v}_{\nu A \rightarrow}+\tilde{v}_{\nu A \leftarrow .}
$$

При помощи этих равенств можно получить соотношения между $u$ и $v$ в окончательном виде:

$$
\begin{aligned}
& \tilde{u}_{\nu \rightarrow}^{(A)}=\frac{1}{2}\left(\tilde{v}_{\nu A \rightarrow}\left(\frac{k_{1 A}+k_{10}}{k_{10}}\right)-\tilde{v}_{\nu A \leftarrow}\left(\frac{k_{1 A}-k_{10}}{k_{10}}\right)\right), \\
& \tilde{u}_{\nu \leftarrow}^{(A)}=\frac{1}{2}\left(-\tilde{v}_{\nu A \rightarrow}\left(\frac{k_{1 A}-k_{10}}{k_{10}}\right)+\tilde{v}_{\nu A \leftarrow}\left(\frac{k_{1 A}+k_{10}}{k_{10}}\right)\right) .
\end{aligned}
$$

2.1. Выход частицы в вакуум. Пусть частица распространяется из левого полупространства в правое. В этом случае нужно положить $\tilde{u}_{\mu \leftarrow}=0$ и, следовательно,

$$
\tilde{u}_{\nu \rightarrow}^{(\mathrm{L})}=\tilde{v}_{\nu \mathrm{L} \rightarrow}-\tilde{v}_{\nu \mathrm{L} \leftarrow}, \quad \tilde{u}_{\nu \rightarrow}^{( \pm)}=\frac{\left(\tilde{v}_{\nu \pm \rightarrow}-\tilde{v}_{\nu \pm \leftarrow}\right) k_{1 \pm}}{k_{10}}
$$


для $\nu=0,2,3$. Используя формулу (19), находим, какая часть отразилась:

$$
\tilde{v}_{\nu \leftarrow}=0, \quad \tilde{v}_{\nu \pm \leftarrow}=\frac{k_{1 \pm}-k_{10}}{k_{1 \pm}+k_{10}} \tilde{v}_{\nu \pm \rightarrow,},
$$

а какая прошла через границу:

$$
\tilde{u}_{\nu \rightarrow}^{(\mathrm{L})}=\tilde{v}_{\nu \mathrm{L} \rightarrow,} \quad \tilde{u}_{\nu \rightarrow}^{( \pm)}=\frac{2 k_{1 \pm}}{k_{10}+k_{1 \pm}} \tilde{v}_{\nu \pm \rightarrow} .
$$

Мы можем выписать дисперсионные соотношения в терминах инвариантной массы $M^{2}=k_{\mu} k^{\mu}=m^{2} \mp \zeta_{x} \sqrt{\omega^{2}-k_{\perp}^{2}}$. В случае дилептонного распада частицы в среде именно инвариантная масса лептонной пары является наблюдаемой величиной. Выражая $\omega$ через $M$, получаем

$$
k_{1 \mathrm{~L}}=\sqrt{\frac{\left(M^{2}-m^{2}\right)^{2}}{\zeta^{2}}-m^{2}}, \quad k_{1 \pm}=\sqrt{\frac{\left(M^{2}-m^{2}\right)^{2}}{\zeta^{2}}-M^{2}} .
$$

В этих терминах дисперсионные законы для поляризаций + и - совпадают по форме, но разница между соответствующими выражениями заключается в области определения: для случая, когда $A=+$ инвариантная масса удовлетворяет условию $M^{2}<\left(\sqrt{m^{2}+\zeta^{2} / 4}-\zeta / 2\right)^{2}$, в то время как при $M^{2}>\left(\sqrt{m^{2}+\zeta^{2} / 4}+\zeta / 2\right)^{2}$ мы имеем дело с поляризацией $A=-$. Следует отметить, что в случае поляризации + квадрат инвариантной массы может принимать отрицательные значения. Так, например, в случае безмассовой частицы для положительно поляризованного фотона $M^{2}<0$. Тем не менее на всем множестве допустимых значений инвариантной массы коэффициент отражения может быть записан в виде

$$
k_{\mathrm{ref}}=\left|\frac{\sqrt{\left(M^{2}-m^{2}\right)^{2} / \zeta^{2}-M^{2}}-\sqrt{\left(M^{2}-m^{2}\right)^{2} / \zeta^{2}-m^{2}}}{\sqrt{\left(M^{2}-m^{2}\right)^{2} / \zeta^{2}-M^{2}}+\sqrt{\left(M^{2}-m^{2}\right)^{2} / \zeta^{2}-m^{2}}}\right| .
$$

Такая зависимость от инвариантной массы позволяет построить график $k_{\mathrm{ref}}\left(M^{2}\right)$. На рис. 1 приведен график для случая выхода фотона из среды с нарушением четности. Как можно видеть из рис. 1, в случае пространственного вектора ЧС и пространственной границы при $\left|M^{2}\right| \gg \zeta^{2}$ большинство фотонов выходит из среды с нарушенной четностью, в то время как значительная часть фотонов с $M^{2} \sim \zeta^{2}$ для поляризации - и с $\left|M^{2}\right| \ll \zeta^{2}$ для поляризации + отражается от границы и не выходит из среды.

2.2. Частица, влетающая в пространство с нарушением четности. Не менее интересно рассмотреть случай вхождения частицы в область с нарушением четности, когда частица движется из правого полупространства в левое. Соответственно надо положить $\tilde{v}_{\mu A \rightarrow}=0$. Из соотношений $(21)$ получаем

$$
\tilde{u}_{\nu \rightarrow}^{(A)}=\frac{k_{10}-k_{1 A}}{k_{10}+k_{1 A}} \tilde{u}_{\nu \leftarrow}^{(A)}, \quad \tilde{v}_{\nu A \leftarrow}=\frac{2 k_{10}}{k_{10}+k_{1 A}} \tilde{u}_{\nu \leftarrow}^{(A)} .
$$




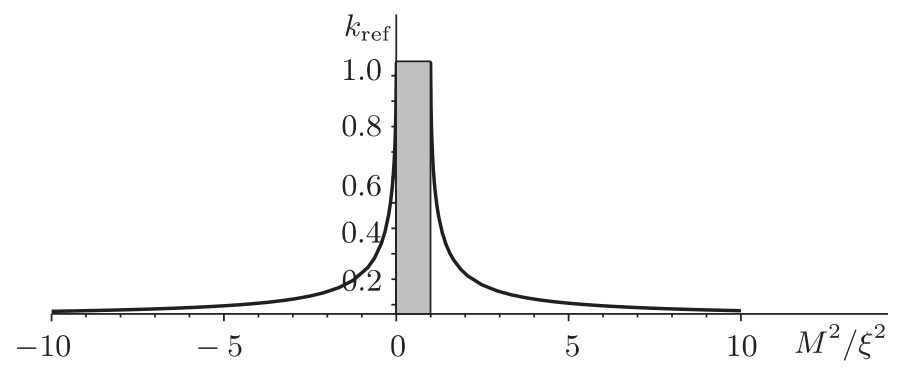

Рис. 1. Коэффициент отражения от границы для фотонов, вылетающих из среды с нарушенной четностью. Заштрихована кинематически запрещенная область значений инвариантной массы.

Единственное, что нам неизвестно в рассматриваемой задаче, это соотношения между $\tilde{u}_{\nu \leftarrow}^{(+)}, \tilde{u}_{\nu \leftarrow}^{(-)}, \tilde{u}_{\nu \leftarrow}^{(\mathrm{L})}$. Мы можем найти их, используя формулы $(15),(27)$ и все амплитуды $\tilde{u}_{\nu \leftarrow}$ падающей волны. Ниже представлены выражения для всех компонент:

$$
\begin{aligned}
& \tilde{u}_{0 \leftarrow}^{(\mathrm{L})}=\frac{\omega^{2}}{\omega^{2}-k_{\perp}^{2}} \tilde{u}_{0 \leftarrow}+\frac{\omega k_{3}}{\omega^{2}-k_{\perp}^{2}} \tilde{u}_{3 \leftarrow}+\frac{\omega k_{2}}{\omega^{2}-k_{\perp}^{2}} \tilde{u}_{2 \leftarrow}, \\
& \tilde{u}_{0 \leftarrow}^{( \pm)}=-\frac{k_{\perp}^{2}}{2\left(\omega^{2}-k_{\perp}^{2}\right)} \tilde{u}_{0 \leftarrow}-\frac{\omega k_{3} \mp i k_{2} \sqrt{\omega^{2}-k_{\perp}^{2}}}{2\left(\omega^{2}-k_{\perp}^{2}\right)} \tilde{u}_{3 \leftarrow}- \\
& -\frac{\omega k_{2} \pm i k_{3} \sqrt{\omega^{2}-k_{\perp}^{2}}}{2\left(\omega^{2}-k_{\perp}^{2}\right)} \tilde{u}_{2 \leftarrow ;} \\
& \tilde{u}_{2 \leftarrow}^{(\mathrm{L})}=-\frac{k_{2}^{2}}{\omega^{2}-k_{\perp}^{2}} \tilde{u}_{2 \leftarrow}-\frac{\omega k_{2}}{\omega^{2}-k_{\perp}^{2}} \tilde{u}_{0 \leftarrow}-\frac{k_{2} k_{3}}{\omega^{2}-k_{\perp}^{2}} \tilde{u}_{3 \leftarrow}, \\
& \tilde{u}_{2 \leftarrow}^{( \pm)}=\frac{\omega^{2}-k_{3}^{2}}{2\left(\omega^{2}-k_{\perp}^{2}\right)} \tilde{u}_{2 \leftarrow}+\frac{\omega k_{2} \mp i k_{3} \sqrt{\omega^{2}-k_{\perp}^{2}}}{2\left(\omega^{2}-k_{\perp}^{2}\right)} \tilde{u}_{0 \leftarrow}+ \\
& +\frac{k_{2} k_{3} \mp i \omega \sqrt{\omega^{2}-k_{\perp}^{2}}}{2\left(\omega^{2}-k_{\perp}^{2}\right)} \tilde{u}_{3 \leftarrow ;} \\
& \tilde{u}_{3 \leftarrow}^{(\mathrm{L})}=-\frac{k_{3}^{2}}{\omega^{2}-k_{\perp}^{2}} \tilde{u}_{3 \leftarrow}-\frac{\omega k_{3}}{\omega^{2}-k_{\perp}^{2}} \tilde{u}_{0 \leftarrow}-\frac{k_{2} k_{3}}{\omega^{2}-k_{\perp}^{2}} \tilde{u}_{2 \leftarrow}, \\
& \tilde{u}_{3 \leftarrow}^{( \pm)}=\frac{\omega^{2}-k_{2}^{2}}{2\left(\omega^{2}-k_{\perp}^{2}\right)} \tilde{u}_{3 \leftarrow}+\frac{\omega k_{3} \pm i k_{2} \sqrt{\omega^{2}-k_{\perp}^{2}}}{2\left(\omega^{2}-k_{\perp}^{2}\right)} \tilde{u}_{0 \leftarrow}+ \\
& +\frac{k_{2} k_{3} \pm i \omega \sqrt{\omega^{2}-k_{\perp}^{2}}}{2\left(\omega^{2}-k_{\perp}^{2}\right)} \tilde{u}_{2 \leftarrow} .
\end{aligned}
$$

Теперь, используя приведенные выше соотношения, мы можем найти, какая часть фотонов пройдет через границу, а какая отразится. У фотона имеются две поперечные поляризации. Решение в правом полупространстве $\left(x_{1}>0\right)$ найдем, используя переменные $\hat{k}$ и $\hat{x}$ :

$$
A^{\mu}(x)=\int d \hat{k} \theta\left(\omega^{2}-k_{\perp}^{2}\right) \sum_{r=1}^{2}\left[a_{\hat{k}, r} u_{\hat{k}, r}^{\mu}(x)+a_{\hat{k}, r}^{*} u_{\hat{k}, r}^{\mu *}(x)\right],
$$




$$
u_{\hat{k}, r}^{\nu}(x)=\left[(2 \pi)^{3} 2 k_{10}\right]^{-1 / 2} e_{r}^{\nu}(\hat{k}) e^{i k_{10} x_{1}+i \hat{k} \cdot \hat{x}}, \quad r=1,2 .
$$

Два линейных поперечных вектора поляризации ортонормированы и на массовой поверхности $k^{2}=0$ удовлетворяют условиям

$$
k^{\mu} e_{\mu}^{i}(\hat{k})=0, \quad g^{\mu \nu} e_{\mu}^{i}(\hat{k}) e_{\nu}^{j}(\hat{k})=\delta^{i j} .
$$

Не умаляя общности, зафиксируем поляризации:

$$
e_{\mu}^{1}(\hat{k})=\left(0,0, \frac{k_{3}}{\left|k_{\perp}\right|},-\frac{k_{2}}{\left|k_{\perp}\right|}\right), \quad e_{\mu}^{2}(\hat{k})=\frac{i}{\left|k_{\perp}\right| \sqrt{\omega^{2}-k_{\perp}^{2}}}\left(k_{\perp}^{2}, 0, \omega k_{2}, \omega k_{3}\right) .
$$

Рассмотрим ситуацию, когда пучок поляризованных фотонов с поляризацией $e^{1}(\hat{k})$ налетает на границу из вакуума:

$$
\hat{u}_{\leftarrow}(\hat{k})=a(\hat{k})\left|k_{\perp}\right| \hat{e}^{1}(\hat{k}) .
$$

При помощи уравнений (28)-(30) находим, что отраженная часть имеет вид

$$
\begin{gathered}
\hat{u}_{\rightarrow}(\hat{k})=a(\hat{k}) \frac{\left|k_{\perp}\right|}{2}\left[\left(\frac{k_{10}-k_{1-}}{k_{10}+k_{1-}}+\frac{k_{10}-k_{1+}}{k_{10}+k_{1+}}\right) \hat{e}^{1}(\hat{k})+\right. \\
\left.+\left(\frac{k_{10}-k_{1-}}{k_{10}+k_{1-}}-\frac{k_{10}-k_{1+}}{k_{10}+k_{1+}}\right) \hat{e}^{2}(\hat{k})\right] .
\end{gathered}
$$

Таким образом, в данном случае мы можем определить два коэффициента отражения

$$
\begin{aligned}
& k_{\mathrm{ref}}^{e^{1} \rightarrow e^{1}}=\left|\frac{1}{2}\left(\frac{1-\sqrt{1-\zeta / k_{1}}}{1+\sqrt{1-\zeta / k_{1}}}+\frac{1-\sqrt{1+\zeta / k_{1}}}{1+\sqrt{1+\zeta / k_{1}}}\right)\right|, \\
& k_{\mathrm{ref}}^{e^{1} \rightarrow e^{2}}=\left|\frac{1}{2}\left(\frac{1-\sqrt{1-\zeta / k_{1}}}{1+\sqrt{1-\zeta / k_{1}}}-\frac{1-\sqrt{1+\zeta / k_{1}}}{1+\sqrt{1+\zeta / k_{1}}}\right)\right|,
\end{aligned}
$$

используя выражения для $k_{1+}, k_{1-}$ и принимая во внимание тот факт, что фотоны, прилетающие из вакуума, находятся на массовой поверхности $\left(k_{1}=k_{10}\right)$.

При значениях $k_{1}<\zeta$ фотоны не могут проникать в среду с нарушенной четностью (это запрещено кинематически). Однако начиная с $k_{1}=\zeta$ фотоны уже могут пересекать границу, поэтому на рис. 2 мы видим излом кривых в точке $\zeta$. Таким образом, фотоны с малыми энергиями полностью отражаются от границы; при повышении энергии некоторая часть при отражении будет менять поляризацию, и лишь при достижении $k_{1}=\zeta$ какая-то часть фотонов может проходить в среду.

Составим поляризации (комбинации $e^{1}$ и $e^{2}$ ) так, чтобы они при отражении от границы в вакуум не смешивались:

$$
e^{\mathrm{L}}=\frac{1}{\sqrt{2}}\left(e^{1}+e^{2}\right), \quad e^{\mathrm{R}}=\frac{1}{\sqrt{2}}\left(e^{1}-e^{2}\right) .
$$

Для получившихся круговых поляризаций определим коэффициенты отражения:

$$
k_{\mathrm{ref}}^{\mathrm{L}}=\left|\frac{1-\sqrt{1-\zeta / k_{1}}}{1+\sqrt{1-\zeta / k_{1}}}\right|, \quad k_{\mathrm{ref}}^{\mathrm{R}}=\left|\frac{1-\sqrt{1+\zeta / k_{1}}}{1+\sqrt{1+\zeta / k_{1}}}\right| .
$$


Следует также обратить внимание на то, что в случае отражения круговые поляризации меняют фазу, так как

$$
e^{\mathrm{L}} \rightarrow \frac{1-\sqrt{1-\zeta / k_{1}}}{1+\sqrt{1-\zeta / k_{1}}} e^{\mathrm{L}}, \quad e^{\mathrm{R}} \rightarrow \frac{1-\sqrt{1+\zeta / k_{1}}}{1+\sqrt{1+\zeta / k_{1}}} e^{\mathrm{R}} .
$$

Если $k_{1}<\zeta$, то при отражении от границы левая поляризация меняет фазу на $\operatorname{Arg}\left[\left(1-\sqrt{1-\zeta / k_{1}}\right) /\left(1+\sqrt{1-\zeta / k_{1}}\right)\right]$. Множитель при $e^{\mathrm{R}}$ на всей области определения $k_{1}>0$ является отрицательным числом, т. е. изменение фазы равно $\pi$.

\section{3. ВРЕМЕНИПОДОБНЫЙ ВЕКТОР ЧС И ПРОСТРАНСТВЕННАЯ ГРАНИЦА}

Случай времениподобного вектора ЧС и пространственноподобной границы $\zeta_{\mu}=$ $\left(\zeta \theta\left(-x_{1}\right), 0,0,0\right)$ может быть полезен для описания процессов, происходящих при столкновении тяжелых ионов, так как позволяет понять, что происходит с частицами внутри файербола. Как и в случае пространственного вектора ЧС, можно найти решения для векторного поля $A$ в виде (11), (13). В этом случае дисперсионные соотношения меняют вид:

$$
\begin{aligned}
& k_{1 \mathrm{~L}}=k_{10}=\sqrt{\omega^{2}-m^{2}-k_{\perp}^{2}}, \\
& k_{1 \pm}=\sqrt{\omega^{2}-m^{2}-k_{\perp}^{2}+\frac{\zeta^{2}}{2} \mp \zeta \sqrt{\omega^{2}-m^{2}+\frac{\zeta^{2}}{4}}} .
\end{aligned}
$$

Как и для пространственноподобного вектора ЧС, мы можем найти соотношения между коэффициентами $\tilde{v}_{i A \rightleftarrows}$ :

$$
\begin{aligned}
& \tilde{v}_{2 A \rightleftarrows}=\frac{-i \zeta k_{1 A}\left(k_{1 A}^{2}-k_{10}^{2}\right)-\zeta^{2} k_{2} k_{3}}{\left(k_{1 A}^{2}-k_{10}^{2}\right)^{2}-\zeta^{2} k_{3}^{2}} \tilde{v}_{3 A \rightleftarrows} \equiv C_{2 A} \tilde{v}_{3 A \rightleftarrows}, \\
& \tilde{v}_{1 A \rightleftarrows}=\frac{-i \zeta k_{2}\left(k_{1 A}^{2}-k_{10}^{2}\right)-\zeta^{2} k_{3} k_{1 A}}{\left(k_{1 A}^{2}-k_{10}^{2}\right)^{2}-\zeta^{2} k_{3}^{2}} \tilde{v}_{3 A \rightleftarrows} .
\end{aligned}
$$

Теперь рассмотрим систему (4) и воспользуемся преобразованием Фурье по переменным $x_{0}, x_{2}, x_{3}$. Как и прежде, условия сшивки, которые получаются из интегрирования системы уравнений по бесконечно малому интервалу $(-\varepsilon, \varepsilon)$, диктуют непрерывность всех пространственных компонент векторного потенциала. Используя этот факт, мы можем написать коэффициенты отражения для каждой поляризации в случае выхода частиц из среды с нарушенной четностью:

$$
k_{\mathrm{ref}}=\left|\frac{k_{1 A}-k_{10}}{k_{1 A}+k_{10}}\right| \text {. }
$$

График для коэффициента отражения построим в зависимости от поперечного импульса $k_{\perp}$ и инвариантной массы $M^{2}=k_{\mu} k^{\mu}$ (см. рис. 3). Представим дисперсионные соотношения в терминах инвариантной массы:

$$
k_{1 A}^{2}=\frac{\left(M^{2}-m^{2}\right)^{2}}{\zeta^{2}}-k_{\perp}^{2}, \quad k_{10}^{2}=\frac{\left(M^{2}-m^{2}\right)^{2}}{\zeta^{2}}+\left(M^{2}-m^{2}\right)-k_{\perp}^{2} .
$$




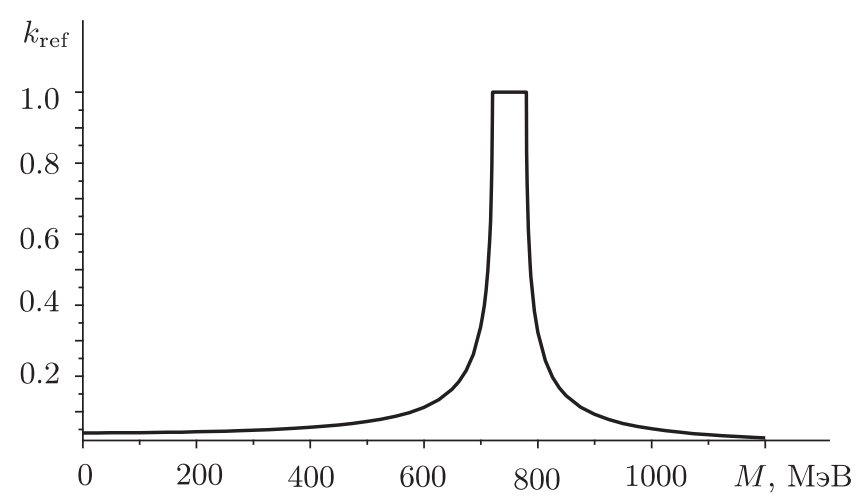

Рис. 3. Коэффициент отражения векторного мезона, летящего перпендикулярно границе. Для векторного мезона полагаем $\zeta=300 \mathrm{MэВ.}$

Дисперсионные соотношения в этих переменных опять одинаковы для поперечных поляризаций, однако их области определения различны. Смена поляризаций происходит в точке $M_{0}^{2}=m^{2}-\zeta^{2} / 4$. Это означает, что при $M<M_{0}$ мы имеем поляризацию -, а при $M>M_{0}$ мы имеем поляризацию + . Кроме того, должно быть выполнено условие, возникающее из кинематических соображений: $\left|k_{\perp}\right| \leqslant\left|M^{2}-m^{2}\right| / \zeta$. Используя все это, мы можем записать коэффициент отражения в виде

$$
k_{\mathrm{ref}}=\left|\frac{\sqrt{\left(M^{2}-m^{2}\right)^{2} / \zeta^{2}-k_{\perp}^{2}}-\sqrt{\left(M^{2}-m^{2}\right)^{2} / \zeta^{2}+\left(M^{2}-m^{2}\right)-k_{\perp}^{2}}}{\sqrt{\left(M^{2}-m^{2}\right)^{2} / \zeta^{2}-k_{\perp}^{2}}+\sqrt{\left(M^{2}-m^{2}\right)^{2} / \zeta^{2}+\left(M^{2}-m^{2}\right)-k_{\perp}^{2}}}\right| .
$$

Вообще говоря, $k_{\text {ref }}$ зависит от $M$ и $k_{\perp}$. Мы приведем здесь график для $\omega$-мезона, который летит перпендикулярно границе: в случае $k_{\perp}=0$. В этом случае для $m^{2}-\zeta^{2}<M^{2}<m^{2}$ коэффициент отражения равен единице.

Как видно из графика, при некоторых инвариантных массах (720-780 МэВ) родившийся в файерболе векторный мезон не может выйти за его пределы, так как испытывает полное внутреннее отражение.

Однако рассмотренный выше выбор вектора ЧС как $\zeta_{\mu}=\left(\zeta \theta\left(-x_{1}\right), 0,0,0\right)$ не обеспечивает калибровочную инвариантность, поскольку при калибровочных преобразованиях не сохраняется функционал

$$
\int d^{3} x \zeta_{\mu} A_{\nu} \partial_{\rho} A_{\sigma} \varepsilon^{\mu \nu \rho \sigma}
$$

Калибровочную инвариантность удается восстановить после добавления еще одной компоненты в вектор ЧС:

$$
\zeta_{\mu}=\left(\zeta \theta\left(-x_{1}\right), \zeta_{x}\left(x_{1}, t\right), 0,0\right), \quad \partial_{1} \zeta_{0}=\partial_{0} \zeta_{1}
$$

Простейшее решение этого дифференциального уравнения есть $\zeta_{x}=-\zeta t \delta\left(x_{1}\right)$. Очевидно, что внутри области с нарушенной пространственной четностью решения уравнений движения останутся прежними. 
Рассмотрим систему (4) с модифицированным вектором ЧС (48) и сделаем преобразование Фурье по переменным $x_{0}, x_{2}, x_{3}$ :

$$
\begin{aligned}
&\left(-\omega^{2}+m^{2}+k_{\perp}^{2}\right) \tilde{A}_{0}-\partial_{1}^{2} \tilde{A}_{0}=-\zeta \partial_{\omega}\left(k_{3} \tilde{A}_{2}-k_{2} \tilde{A}_{3}\right) \delta\left(x_{1}\right), \\
&\left(-\omega^{2}+m^{2}+k_{\perp}^{2}\right) \tilde{A}_{1}-\partial_{1}^{2} \tilde{A}_{1}=-i \zeta \theta\left(-x_{1}\right)\left(k_{3} \tilde{A}_{2}-k_{2} \tilde{A}_{3}\right), \\
&\left(-\omega^{2}+m^{2}+k_{\perp}^{2}\right) \tilde{A}_{2}-\partial_{1}^{2} \tilde{A}_{2}= \zeta \theta\left(-x_{1}\right)\left(i k_{3} \tilde{A}_{1}+\partial_{1} \tilde{A}_{3}\right)- \\
&-\zeta \partial_{\omega}\left(k_{3} \tilde{A}_{0}+\omega \tilde{A}_{3}\right) \delta\left(x_{1}\right), \\
&\left(-\omega^{2}+m^{2}+k_{\perp}^{2}\right) \tilde{A}_{3}-\partial_{1}^{2} \tilde{A}_{3}=\zeta \theta\left(-x_{1}\right)\left(-i k_{2} \tilde{A}_{1}-\partial_{1} \tilde{A}_{2}\right)+ \\
&+\zeta \partial_{\omega}\left(k_{2} \tilde{A}_{0}+\omega \tilde{A}_{2}\right) \delta\left(x_{1}\right) .
\end{aligned}
$$

Интегрируя эту систему по переменной $x_{1}$ от $-\varepsilon$ до $\varepsilon$, находим новые условия сшивки для поперечных поляризаций:

$$
\begin{aligned}
\left(\tilde{u}_{0 \leftarrow}^{( \pm)}-\tilde{v}_{0 \leftarrow}^{( \pm)}\right) & =\frac{i \zeta}{2 k_{10} \omega^{2}} C_{ \pm}\left(k_{2}, k_{3}\right), \\
\partial_{1} \tilde{A}_{1} & \text { непрерывна, } \\
\tilde{u}_{0 \leftarrow}^{( \pm)}+\tilde{u}_{0 \rightarrow}^{( \pm)} & =\frac{C_{ \pm}\left(k_{2}, k_{3}\right)}{k_{3}\left(k_{2}-k_{3} C_{2 A}\right)}+C_{u \pm}\left(k_{2}, k_{3}\right), \\
\tilde{v}_{3 \pm \rightarrow}+\tilde{v}_{3 \pm \leftarrow} & =\frac{C_{ \pm}\left(k_{2}, k_{3}\right)}{\omega\left(k_{2}-k_{3} C_{2 A}\right)},
\end{aligned}
$$

и для продольной поляризации:

$$
\begin{aligned}
& \tilde{u}_{0 \leftarrow}^{(\mathrm{L})}=\tilde{v}_{0 \leftarrow}^{(\mathrm{L})}, \\
& \partial_{1} \tilde{A}_{1} \text { непрерывна, } \\
& \tilde{u}_{0 \leftarrow}^{(\mathrm{L})}+\tilde{u}_{0 \rightarrow}^{(\mathrm{L})}=C_{u \mathrm{~L}}\left(k_{2}, k_{3}\right), \\
& \tilde{v}_{3 \mathrm{~L} \rightarrow}+\tilde{v}_{3 \mathrm{~L} \leftarrow}=\frac{C_{\mathrm{L}}\left(k_{2}, k_{3}\right)}{\omega},
\end{aligned}
$$

где $C_{ \pm}\left(k_{2}, k_{3}\right), C_{\mathrm{L}}\left(k_{2}, k_{3}\right), C_{u \pm}\left(k_{2}, k_{3}\right)$ и $C_{u \mathrm{~L}}\left(k_{2}, k_{3}\right)$ - произвольные функции переменных $k_{2}$ и $k_{3}$.

Условие непрерывность $\partial_{1} \tilde{A}_{1}$ и соотношения (43) обеспечивают непрерывность всех пространственных компонент векторного потенциала. Учитывая это, мы можем использовать полученные выше результаты. Таким образом, выражение (46) для коэффициента отражения сохраняется, и эффекты, связанные с полным (или частичным) внутренним отражением, проявляются в той же форме.

\section{4. ЗАКЛЮЧЕНИЕ}

В настоящей работе изучена модель физических явлений, связанных с распространением фотонов и векторных мезонов между областью с нарушением пространственной четности и вакуумом. Основные результаты получены для пространственноподобного вектора ЧС. Представлены соотношения, с помощью которых можно рассчитывать прохождение или отражение входящих или выходящих из области частиц с любой поляризацией. В частности, доказано, что поперечные поляризации испытывают сильное отражение вплоть до полного внутреннего отражения при 
определенных частотах. Аналогичные соотношения найдены для времениподобного вектора ЧС, но в случае пространственноподобной границы, когда возникают проблемы с калибровочной инвариантностью. Показано, что упомянутые проблемы не сказываются на коэффициентах отражения и прохождения, которые и являются в данном случае наблюдаемыми величинами. Кроме того, для пространственноподобного вектора ЧС обнаружено, что при облучении фотонами среды с нарушенной четностью может происходить дополнительное вращение круговых поляризаций при отражении от границы. Таким образом, влияние границы между областью с нарушением четности и вакуумом на ширину распада фотонов и векторных мезонов представляет собой интересную задачу, заслуживающую дальнейшего исследования для предсказаний эффектов нарушения четности в ядерных средах и в астрофизике плотных звезд.

Благодарности. Работа поддержана РФФИ (грант № 13-02-00127), а также Санкт-Петербургским государственным университетом (проект № 11.38.660.2013). C. С. Колеватов благодарит фонд некоммерческих программ "Династия" за финансовую поддержку.

\section{Список литературы}

[1] S. M. Carroll, G. B. Field, R. Jackiw, Phys. Rev. D, 41:4 (1990), 1231-1240.

[2] A. A. Andrianov, R. Soldati, Phys. Rev. D, 51:10 (1995), 5961-5964, arXiv: hep-th/9405147.

[3] V.A. Kostelecký, M. Mewes, Phys. Rev. D, 66:5 (2002), 056005, 24 pp., arXiv: hep-ph/0205211.

[4] G. M. Shore, Contemp. Phys., 44:6 (2003), 503-521, arXiv: gr-qc/0304059.

[5] N. Arkani-Hamed, H.-C. Cheng, M. A. Luty, S. Mukohyama, JHEP, 05 (2004), 074, 36 pp.; H.-C. Cheng, M. A. Luty, S. Mukohyama, J. Thaler, JHEP, 05 (2006), 076, arXiv: hep-th/0603010.

[6] F. E. Schunck, E. W. Mielke, Class. Quantum Grav., 20:20 (2003), R301-R356.

[7] A. A. Andrianov, D. Espriu, P. Giacconi, R. Soldati, JHEP, 09 (2009), 057, 16 pp., arXiv: 0907.3709.

[8] A. A. Andrianov, D. Espriu, F. Mescia, A. Renau, Phys. Lett. B, 684:2-3 (2010), 101-105, arXiv: 0912.3151.

[9] R. Arnaldi, R. Averbeck, K. Banicz et al. [NA60 Collaboration], Phys. Rev. Lett., 96:16 (2006), 162302, 5 pp., arXiv: nucl-ex/0605007.

[10] A. Adare, S. Afanasiev, C. Aidala et al. [PHENIX Collaboration], Phys. Rev. C, 81:3 (2010), 034911, 56 pp., arXiv: 0912.0244.

[11] A. A. Andrianov, V.A. Andrianov, D. Espriu, X. Plannels, Phys. Lett. B, 710:1 (2012), 230-235, arXiv: 1010.4688.

[12] A. A. Andrianov, D. Espriu, Phys. Lett. B, 663:5 (2008), 450-455, arXiv: 0709.0049; A. A. Andrianov, D. Espriu, V. A. Andrianov, Phys. Lett. B, 678:4 (2009), 416-421, arXiv: 0904.0413.

[13] J. Alfaro, A. A. Andrianov, M. Cambiaso, P. Giacconi, R. Soldati, Internat. J. Modern Phys. A, 25:16 (2010), 3271-3306; Phys. Lett. B, 639:5 (2006), 586-590, arXiv: hep-th/0604164.

[14] A. A. Andrianov, S. S. Kolevatov, R. Soldati, JHEP, 11 (2011), 007, arXiv: 1109.3440. 